Pacific Journal of Mathematics

THE SECOND COUSIN PROBLEM WITH BOUNDED DATA 


\title{
THE SECOND COUSIN PROBLEM WITH BOUNDED DATA
}

\author{
E. L. Stout
}

\begin{abstract}
Given a complex manifold $M$, an open covering $\mathscr{Y} \equiv$ $\left\{V_{\alpha}\right\}_{\alpha \in A}$, and, for each $\alpha \in A$ a function $f_{\alpha}$ holomorphic on $V_{\alpha}$ such that for all $\alpha, \alpha^{\prime} \in A, f_{\alpha} f_{\alpha^{\prime}}^{-1}$ is a zero-free holomorphic function on $V_{\alpha} \cap V_{\alpha^{\prime}}$, the associated second Cousin problem is the problem of showing the existence of a function $F$ holomorphic on $M$ such that for all $\alpha, F f_{\alpha}^{-1}$ is a zero free function holomorphic on $V_{\alpha}$. In the present paper we consider an analogous problem in the case that $M$ is the open unit polycylinder $U^{N}=\left\{\left(z_{1}, \cdots, z_{N}\right) \in \mathbf{C}^{N}:\left|z_{1}\right|<1, \cdots,\left|z_{N}\right|<1\right\}$, that the functions $f_{\alpha}$ are required to be bounded and that the sought function $F$ is also required to be bounded.
\end{abstract}

If $\Omega$ is an open set in $\mathrm{C}^{N}$, we denote by $H^{\infty}[\Omega]$ the algebra of functions bounded and holomorphic in $\Omega$. In the first section of the present paper we will establish the following result.

THEOREM I.1. Let $\mathscr{Y}^{-}=\left\{V_{\alpha \in A}\right\}_{\alpha \in}$ be an open covering of $\bar{U}^{N}$, the closure of $U^{N}$, and for each $\alpha$, let $f_{\alpha} \in H^{\infty}\left[V_{\alpha} \cap U^{N}\right]$. If for all $\alpha, \beta \in A, f_{\alpha} f_{\beta}^{-1}$ is an invertible element of $H^{\infty}\left[V_{\alpha} \cap V_{\beta} \cap U^{N}\right]$, then there exists $F \in H^{\infty}\left[U^{N}\right]$ such that for all $\alpha, F f_{\alpha}^{-1}$ is an invertible element of $H^{\infty}\left[V_{\alpha} \cap U^{N}\right]$.

An analogous theorem is valid in the case that we consider functions with continuous boundary values; it is essentially contained in the thesis of Douady. In $\S I I$ we consider this result briefly and give an application of it to an interpolation theorem.

I. Proof of theorem I.1. A remark concerning the statement may be in order. Although the functions $f_{\alpha}$ are only defined on subsets of $U^{N}$ and although we seek a function $F$ defined only in $U^{N}$, it seems necessary to require that $\mathscr{V}$ be an open covering of $\bar{U}^{N}$ even if we require $\mathscr{V}$ to be finite. A relevant example, with $\mathscr{V}^{-}$an open cover of $U=U^{1}$ which consists of two sets is as follows. Let $\lambda$ be an arc with and points 1 and -1 which is contained, except for its end points, in the lower half of the open unit disc. If $\lambda$ approaches the real axis with sufficient rapidity, the sequence $\{1-1 / n\}_{n=1}^{\infty}$ will be the zero set of a function $f_{1}$ holomorphic and bounded on the set $V_{1}=\{z \in U: z$ lies above $\lambda\}$. We can choose $f_{1}$ to be continuous on $V_{1} \cup$ (interior $\lambda$ ) and of modulus 1 on $\lambda$. We take for $V_{2}$ the part of $U$ lying on and below $\lambda$ together with the set $\left\{z \in V_{1}:\left|f_{1}(z)\right|>1 / 2\right\}$. 
Define $f_{2}$ on $V_{2}$ to be identically 1 . Then $\left\{V_{1}, V_{2}\right\}$ is an open cover for $U$. On $V_{1} \cap V_{2}, f_{1} f_{2}^{-1}$ is bounded and bounded away from 0 . But since the sequence $\{1-1 / n\}$ does not satisfy the Blaschke condition, it is not the zero set of any $f \in H^{\infty}[U]$.

The proof of the theorem depends on the following lemma.

LEMMA 1.2. Let $f=\widetilde{u}+i \widetilde{v}$ be a function holomorphic in $U^{N}$. Let $\lambda_{1}$ and $\lambda_{2}$ be disjoint arcs in the unit circle, and define $V_{j}, j=1,2$, to be the union of $U$, the interior of $\lambda_{j}$, and the exterior, including $\infty$, of the unit disc. If $\tilde{u}$ is bounded we may write, for $z \in U^{N}$, $f(z)=f_{1}(z)+f_{2}(z)$ where $f_{j}$ is holomorphic and has bounded real part in $V_{j} \times U^{N-1}$.

We shall defer the proof of the lemma for the moment and proceed to show how the lemma implies the theorem. It is more convenient to work in the polycube than in the polycylinder. We set

$$
\begin{array}{r}
\Delta^{+}=\left\{z \in \mathbf{C}^{N}: z_{j}=x_{j}+i y_{j},-\frac{1}{2}<\right. \\
x_{1}<1,\left|x_{2}\right|, \cdots,\left|x_{N}\right|, \\
\left.\left|y_{1}\right|, \cdots,\left|y_{N}\right|<1\right\},
\end{array}
$$

and

$$
\Delta^{-}=\left\{z \in \mathbf{C}^{N}:-1<x_{1}<\frac{1}{2},\left|x_{2}\right|, \cdots,\left|x_{N}\right|,\left|y_{1}\right|, \cdots,\left|y_{N}\right|<1\right\} .
$$

Assume given $F$ an invertible element of $H^{\infty}\left[\Delta^{+} \cap \Delta^{-}\right]$. We assert that on $\Delta^{+} \cap \Delta^{-}$,

$$
F=F^{+} F^{-}
$$

where $F^{+}$and $F^{-}$are invertible elements of $H^{\infty}\left[\Delta^{+}\right]$and $H^{\infty}\left[\Delta^{-}\right]$ respectively.

We may write $F=e^{\theta}$ where $G$ is holomorphic in $\Delta^{+} \cap \Delta^{-}$and where $R e G$ is bounded; in general $\operatorname{Im} G$ will not be bounded. The lemma implies that $G=G_{+}+G_{-}$where $G_{+}$and $G_{-}$are holomorphic in $\Delta^{+}$and $\Delta^{-}$respectively and have bounded real parts: This can be proved in the following way.

Let $\alpha$ be a conformal map of $U$ onto $\{z=x+i y:|x|,|y|<1\}$ and $\beta$ one from $U$ onto $\{z=x+i y:|x|<1 / 2,|y|<1\}$. Then the map $\Phi: U^{N} \rightarrow \Delta^{+} \cap \Delta^{-}$given by $\Phi(z)=\left(\beta\left(z_{1}\right), \alpha\left(z_{2}\right), \cdots, \alpha\left(z_{N}\right)\right)$ is an analytic homeomorphism. Let

$$
\begin{aligned}
& \Lambda_{1}=\left\{\frac{1}{2}+i y:|y| \leqq 1\right\}, \\
& \Lambda_{2}=\left\{-\frac{1}{2}+i y:|y| \leqq 1\right\},
\end{aligned}
$$


and let $\lambda_{1}, \lambda_{2}$ be respectively the preimages of $\Lambda_{1}$ and $\Lambda_{2}$ under $\beta$. Let $V_{1}$ and $V_{2}$ be the domains in the Riemann sphere constructed from $\lambda_{1}$ and $\lambda_{2}$ respectively as in the statement of the lemma. Given a point $w,|w|>1$, we define $\beta_{1}(w)$ to be $1-[\beta(1 / \bar{w})]^{-}$. (Here $[\cdot]^{-}$ is used to indicate complex conjugation.) This definition effects a continuation of $\beta$ to a conformal one-to-one mapping of $V_{1}$ into $\mathrm{C}$ in such a way that the range of the continued map, $\beta_{1}$, contains

$$
\left\{z=x+i y:|y|<1,-\frac{1}{2}<x<1\right\} \text {. }
$$

In the same way $\beta$ continues to a conformal map $\beta_{2}$ of $V_{2}$ into $\mathrm{C}$ such that $\beta_{2}\left[V_{2}\right]$ contains

$$
\left\{z=x+i y:|y|<1,-1<x<\frac{1}{2}\right\} \text {. }
$$

Define $\Phi_{j}: V_{j} \times U^{N-1} \rightarrow \mathrm{C}^{N}$ to be the natural continuation of $\Phi$ from $U^{N}$ into the set $V_{j} \times U^{N-1}$. The range of $\Phi_{1}$ contains $\Delta^{+}$, that of $\Phi_{2}, \Delta^{-}$.

Apply the lemma to the function $G \circ \Phi$ to get $G \circ \Phi=G_{1}+G_{2}$ where $G_{j}$ is holomorphic and has bounded real part in $V_{j} \times U^{N-1}$. But then $G_{j} \circ \Phi_{j}^{-1}$ is holomorphic and has bounded real part in $\Delta^{+}$if $j=1$, in $\Delta^{-}$if $j=2$, and we have, since $\Phi_{1}^{-1}=\Phi_{2}^{-1}=\Phi^{-1}$ on $\Delta^{+} \cap \Delta^{-}$, that in $\Delta^{+} \cap \Delta^{-}, G=G_{1} \circ \Phi_{1}^{-1}+G_{2} \circ \Phi_{2}^{-1}$. This is a decomposition of $G$ of the desired kind.

For the function $F_{+}$, we take $\exp \left(G_{1} \circ \Phi_{1}^{-1}\right)$, and for $F_{-}$we take $\exp \left(G_{2} \circ \Phi_{2}^{-1}\right)$. This choice of $F_{+}$and $F_{-}$gives the decomposition of $F$ which we seek.

As soon as we have the decomposition (I.1.1.) for invertible elements of $H^{\infty}\left[\Delta^{+} \cap \Delta^{-}\right]$, the theorem can be established by a patching argument familiar in this context. (See, e.g. [1].) For the rest of the argument, let us set $\Delta_{1}^{1}=\Delta^{+}, \Delta_{1}^{2}=\Delta^{-}$. Suppose given $\mathscr{V}^{-}=\left\{V_{\alpha}\right\}$, an open covering of the closure of the polycube $\Delta=\Delta_{1}^{1} \cup \Delta_{1}^{2}$, and for each $\alpha, f_{\alpha} \in H^{\infty}\left[V_{\alpha} \cap \Delta\right]$ such that $f_{\alpha} f_{\beta}^{-1}$ is invertible in $H^{\infty}\left[\Delta \cap V_{\alpha} \cap V_{\beta}\right]$. Suppose the theorem is false so that for no $F \in H^{\infty}[\Delta]$ is it the case that for all $\alpha, F f_{\alpha}^{-1}$ is invertible in $H^{\infty}\left[V_{\alpha} \cap \Delta\right]$. Then it cannot be that the induced problems on $\Delta_{1}^{1}$ and $\Delta_{1}^{2}$ are both solvable. That is to say, it cannot be the case that there exist $F_{1}$ and $F_{2}$ in $H^{\infty}\left[\Delta_{1}^{1}\right]$ and $H^{\infty}\left[\Delta_{1}^{2}\right]$ respectively such that $F_{j} f_{\alpha}^{-1}$ is, for all $\alpha$, an invertible element of $H^{\infty}\left[V_{\alpha} \cap \Delta_{1}^{j}\right]$.

Suppose that such $F_{1}$ and $F_{2}$ exist. The function $F_{1} F_{2}^{-1}$ is then an invertible element of $H^{\infty}\left[\Delta_{1}^{1} \cap \Delta_{1}^{2}\right]$. Since $\mathscr{Y}$ is an open cover for the closed polycube $\bar{\Delta}$, finitely many of the elements of $\mathscr{V}$, say $V_{1}, \cdots, V_{p}$ cover $\Delta_{1}^{1} \cap \Delta_{1}^{2}$. Thus, there are $\varepsilon$ and $M>0$ such that for 
$j=1,2, \cdots, p,\left|F_{1} f_{j}^{-1}\right|$ and $\left|F_{2} f_{j}^{-1}\right|$ are at least $\varepsilon$ but no more than $M$ on $\Delta_{1}^{1} \cap V_{j}$ and $\Delta_{1}^{2} \cap V_{j}$ respectively. Thus, if $z \in \Delta_{1}^{1} \cap \Delta_{1}^{2}$ so that $z \in V_{j}$, say, then

$$
\left|F_{1}(z) F_{2}(z)^{-1}\right|=\left|\left(F_{1}(z) f_{j}(z)\right)\left(F_{2}(z) f_{j}(z)\right)^{-1}\right|
$$

and this is no more than $M / \varepsilon$ and no less than $\varepsilon / M$. Consequently, the decomposition (I.1.1.) applies to $F_{1} F_{2}^{-1}$ and provides $G_{1}$ and $G_{2}$, invertible elements of $H^{\infty}\left[\Delta_{1}^{1}\right]$ and $H^{\infty}\left[\Delta_{1}^{2}\right]$ respectively, such that on $\Delta_{1}^{1} \cap \Delta_{1}^{2}$ we have

whence

$$
F_{1} F_{2}^{-1}=G_{1} G_{2}
$$

$$
F_{1} G_{1}^{-1}=F_{2} G_{2} .
$$

Thus, we can define $F \in H^{\infty}[\Delta]$ by setting

$$
F(z)= \begin{cases}F_{1}(z) G_{1}^{-1}(z) & z \in \Delta_{1}^{1} \\ F_{2}(z) G_{2}(z) & z \in \Delta_{1}^{2}\end{cases}
$$

Consider now $F f_{\alpha}^{-1}$ on $V_{\alpha}$. On $V_{\alpha} \cap \Delta_{1}^{1}$, this is $\left(F F_{1}^{-1}\right)\left(F_{1} f_{\alpha}^{-1}\right)$ and so is an invertible element of $H^{\infty}\left[V_{\alpha} \cap \Delta_{1}^{1}\right]$ and on $V_{\alpha} \cap \Delta_{1}^{2}$ it is $\left(F F_{2}^{-1}\right)\left(F_{2} f_{\alpha}^{-1}\right)$ and so is invertible in $H^{\infty}\left[V_{\alpha} \cap \Delta_{1}^{2}\right]$. Thus, for all $\alpha, F f_{\alpha}^{-1}$ is invertible in $H^{\infty}\left[V_{\alpha} \cap \Delta\right]$. By hypothesis no such $F$ exists, so we must conclude that either $F_{1}$ or $F_{2}$ does not exist.

Suppose $F_{1}$ does not exist. Set

$$
\begin{aligned}
& \Delta_{2}^{1}=\left\{z \in \Delta_{1}^{1}: \quad-\frac{1}{2}<y_{1}<1\right\} \\
& \Delta_{2}^{2}=\left\{z \in \Delta_{1}^{1}: \quad-1<y_{1}<\frac{1}{2}\right\} .
\end{aligned}
$$

Arguing as in the last paragraph, there do not exist functions $F_{2}^{(1)}$ and $F_{2}^{(2)}$ in $H^{\infty}\left[\Delta_{2}^{1}\right]$ and $H^{\infty}\left[\Delta_{2}^{2}\right]$ respectively such that for all $\alpha, F_{2}^{(j)} f_{\alpha}^{-1}$ is invertible in $H^{\infty}\left[\Delta_{2}^{j}\right], j=1,2$. Thus, the induced problem is not solvable on both $\Delta_{2}^{1}$ and $\Delta_{2}^{2}$.

Iterating this procedure, proceeding cyclicly through the real coordinates of $\mathbf{C}^{N}$ we obtain a nested sequence $\Delta_{1}^{1} \supset \Delta_{2}^{k_{2}} \supset \Delta_{3}^{k_{3}} \supset \cdots$ of cubes with diameter decreasing to zero on none of which we are able to solve the induced problem. This leads to a contradiction, though, for $\mathscr{V}^{-}$is an open cover for the closure of $\Delta$ so for some $N$ and some $\alpha, \Delta_{n}^{k} \subset \subset V_{\alpha}$ if $n \geqq N$, and the function $f_{\alpha}$ is then a solution to the induced problem on $\Delta_{n}^{k_{n}}$ for all $n \geqq N$.

Thus, it remains only to prove the lemma.

Proof of Lemma 1.2. It is convenient to establish at the outset certain notations which will be useful throughout the proof. We will 
denote by $Z^{N}$ the set of $N$-tuples of integers. Let $E=\{n=$ $\left(n_{1}, \cdots, n_{N}\right) \in \boldsymbol{Z}^{N}: n_{1} \geqq 0, \cdots, n_{N} \geqq 0$ or else $\left.n_{1} \leqq 0, \cdots, n_{N} \leqq 0\right\}$. Given $n \in \boldsymbol{Z}^{N}$, let $E^{+}(n)=\left\{m \in \boldsymbol{Z}^{N}: n_{1} \leqq m_{1}, \cdots, n_{N} \leqq m_{N}\right\}, E^{-}(n)=\left\{m \in \boldsymbol{Z}^{N}\right.$ : $\left.n_{1} \geqq m_{1}, \cdots, n_{N} \geqq m_{N}\right\}$. If $z=\left(r_{1} e^{i \theta_{1}}, \cdots, r_{N} e^{i \theta_{N}}\right)$ is in $U^{N}$ and $\varphi=$ $\left(\varphi_{1}, \cdots, \varphi_{N}\right) \in \boldsymbol{R}^{N}$, put

$$
P(z, \varphi)=\sum_{n \in Z^{N}} r_{1}^{\left|n_{1}\right|} \cdots r_{N}^{\left|n_{N}\right|} e^{i n \bullet(\theta-\varphi)}
$$

and

$$
K(z, \varphi)=\sum_{n \in E} r_{1}^{\left|n_{1}\right|} \cdots r_{N}^{\left|n_{N}\right|} e^{i n \bullet(\theta-\varphi)} .
$$

Here we use $n \cdot(\theta-\varphi)$ as abbreviation for $n_{1}\left(\theta_{1}-\varphi\right)+\cdots+n_{N}\left(\theta_{N}-\varphi_{N}\right)$. The kernel $P$ is the $N$ dimensional Poisson kernel. A short calculation shows that

$$
K(z, \varphi)=\operatorname{Re}\left\{\frac{2}{\left(1-z_{1} e^{-i \varphi_{1}}\right) \cdots\left(1-z_{N} e^{-i \varphi_{N}}\right)}\right\}-1 .
$$

A preliminary reduction of the problem seems desirable. The function $f$ of the lemma may be written as the sum $f=f_{0}+f_{1}$ where $f_{0}\left(z_{1}, \cdots, z_{N}\right)=f\left(0, z_{2}, \cdots, z_{N}\right)$ and $f_{1}=f-f_{0}$. The function $f_{0}$ is holomorphic in the product of the Riemann sphere and $U^{N-1}$, and it has bounded real part. Thus, it is enough to prove the lemma with $f$ replaced by $f_{1}$. Let $f_{1}=u+i v$; the function $u$ is bounded. For $0 \leqq \rho_{1}, \cdots, \rho_{N}<1$, we may write

$$
u\left(\rho_{1} e^{i \theta_{1}}, \cdots, \rho_{N} e^{i \theta_{N}}\right)=\sum_{k \in E} \widehat{u}(k) \rho_{1}^{\left|k_{1}\right|} \cdots \rho_{N}^{\left|k_{N}\right|} e^{i k \cdot \theta}
$$

where $\hat{u}(k)$ denotes the $k^{t h}$ Fourier coefficient of the function $u$ :

$$
\hat{u}(k)=\left(\frac{1}{2 \pi}\right)^{N} \int_{-\pi}^{\pi} \cdots \int_{-\pi}^{\pi} u\left(e^{i \varphi_{1}}, \cdots, e^{i \varphi_{N}}\right) e^{-i k \cdot \varphi} d \varphi_{1} \cdots d \varphi_{N} .
$$

Since $u=R e f_{1}$ and since $f_{1}\left(0, z_{2}, \cdots, z_{N}\right)=0$, it follows that $\hat{u}\left(0, k_{2}, \cdots, k_{N}\right)=0$ for all choices of $k_{2}, \cdots, k_{N}$. Thus the summation in (I.2.2) extends over the set $E^{+}(1,0, \cdots, 0) \cup E^{-}(-1,0, \cdots, 0)$.

Let $h$ be a real function of class $\mathscr{C}^{\infty}$ on the circle which vanishes identically on a neighborhood of $\lambda_{1}$ and is identically one on a neighborhood of $\lambda_{2}$. Let $h$ have Fourier expansion $h\left(e^{i \varphi_{1}}\right)=\sum_{n=-\infty}^{\infty} c_{m} e^{i m \varphi_{1}}$. Note that since $h$ is real, $c_{-m}=\bar{c}_{m}$. Define $g_{1}$ and $g_{2}$ by means of

$$
g_{1}(z)=\left(\frac{1}{2 \pi}\right)^{N} \int u(\varphi) h\left(e^{i \varphi_{1}}\right)\left\{\frac{2}{\left(1-z_{1} e^{-i \varphi_{1}}\right) \cdots\left(1-z_{N} e^{-i \varphi_{N}}\right)}-1\right\} d \varphi
$$

and

$$
g_{2}(z)=\left(\frac{1}{2 \pi}\right)^{N} \int u(\varphi)\left\{1-h\left(e^{i \varphi_{1}}\right)\right\}\left\{\frac{2}{\left(1-z_{1} e^{-i \varphi_{1}}\right) \cdots\left(1-z_{N} e^{-\varphi_{N}}\right)}-1\right\} d \varphi .
$$


Here we have written $u(\varphi)$ instead of $u\left(e^{i \varphi_{1}}, \cdots, e^{i \varphi_{N}}\right)$ and $d \varphi$ instead of $d \varphi_{1} \cdots d \varphi_{N}$. The integrals extend over $-\pi \leqq \varphi_{1}, \cdots, \varphi_{N} \leqq \pi$. Since $h$ is identically zero on $\lambda_{1}$, it follows that the function $g_{1}$ is holomorphic in $V_{1} \times U^{N-1}$. Similarly, $g_{2}$ is holomorphic in $V_{2} \times U^{N-1}$. We shall show that $g_{1}$ and $g_{2}$ have bounded real parts. We have $\operatorname{Re} g_{1}$ $+\operatorname{Re} g_{2}=u$ whence for some purely imaginary constant $\gamma, f_{1}=$ $g_{1}+\left(g_{2}+\gamma\right)$ is a decomposition of $f_{1}$ of the kind we seek. It is enough to establish the boundedness of $\operatorname{Re} g_{1}$.

Since $u$ and $h$ are real, we have

$$
\begin{gathered}
\operatorname{Re} g_{1}(z)=\left(\frac{1}{2 \pi}\right)^{N} \int u(\varphi) h\left(e^{i \varphi_{1}}\right) K(z, \varphi) d \varphi \\
=c_{0}\left(\frac{1}{2 \pi}\right)^{N} \int u(\varphi) K(z, \varphi) d \varphi+\sum_{m=1}^{\infty}\left(\frac{1}{2 \pi}\right)^{N} \int u(\varphi)\left[c_{m} e^{i m \varphi_{1}}\right. \\
\left.+\bar{c}_{m} e^{-i m \varphi_{1}}\right] K(z, \varphi) d \varphi .
\end{gathered}
$$

Let $\mu_{m}$ be the measure on $T^{N}$ whose Fourier transform $\hat{\mu}_{m}$ is the characteristic function of $\left\{n \in \boldsymbol{Z}^{N}:-m+1 \leqq n_{1} \leqq-1\right\}$ and let $\nu_{m}$ be such that $\hat{\nu}_{m}$ is the characteristic function of $\left\{n \in \boldsymbol{Z}^{N}: 1 \leqq n_{1} \leqq m-1\right\}$; the measures $\mu_{m}$ and $\nu_{m}$ are of norm no more than $C \log (1+m)$ for some absolute constant $C$. Define $A_{m}$ on $T^{N}$ by

$$
A_{m}(\varphi)=c_{m} e^{i m \varphi_{1}}\left(u-u * \mu_{m}\right)(\varphi)+\bar{c}_{m} e^{-i m \varphi_{1}}\left(u-u * \nu_{m}\right)(\varphi)
$$

where $u * \mu$ denotes the convolution of the function $u$ and the measure $\mu$. The Fourier series of the function $u$ is

$$
\sum\left\{\widehat{u}(k) e^{i k \cdot \varphi}: k \in E^{+}(1,0, \cdots, 0) \cup E^{-}(-1,0, \cdots, 0)\right\}
$$

so that of $A_{m}$ is

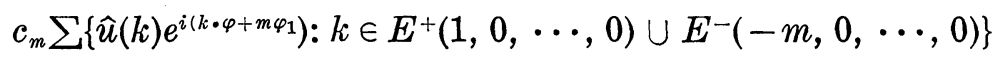

$$
\begin{aligned}
& +\bar{c}_{m} \sum\left\{\hat{u}(k) e^{i\left(k \cdot \varphi-m \varphi_{1}\right)}: k \in E^{+}(m, 0, \cdots, 0) \cup E^{-}(-1,0, \cdots, 0)\right\} \text {. }
\end{aligned}
$$

Since $\hat{u}=0$ off $E^{+}(1,0, \cdots, 0) \cup E^{-}(-1,0, \cdots, 0)$, it follows from the definition of $K(z, \varphi)$ and $P(z, \varphi)$, that

$$
\begin{gathered}
\left(\frac{1}{2 \pi}\right)^{N} \int u(\varphi)\left[c_{m} e^{i m \varphi_{1}}+\bar{c}_{m} e^{-i m \varphi_{1}}\right] K(z, \varphi) d \varphi \\
=\left(\frac{1}{2 \pi}\right)^{N} \int A_{m}(\varphi) P(z, \varphi) d \varphi .
\end{gathered}
$$

Also, $\quad\left(\frac{1}{2 \pi}\right)^{N} \int u(\varphi) K(z, \varphi)=\left(\frac{1}{2 \pi}\right)^{N} \int u(\varphi) P(z, \varphi) d \varphi$.

Thus the series (I.2.3) is bounded term by term by the series $2 C\|u\|_{\infty} \sum_{m=0}^{\infty}\left|c_{m}\right|(1+\log (1+m))$ provided $z \in U^{N}$. Since $c_{m}=O\left(m^{-2}\right)$, 
it follows that $\operatorname{Re} g_{1}$ is bounded in $U^{N}$.

We must show that $\operatorname{Re} g_{1}\left(z_{1}, z_{2}, \cdots, z_{N}\right)$ is bounded for $\left|z_{1}\right|>1$, $\left|z_{2}\right|, \cdots,\left|z_{N}\right|<1$. $^{1)} \quad$ If $\left|z_{1}\right|<1$, set $z_{1}^{*}=1 / \bar{z}_{1}$ so $\left|z_{1}^{*}\right|>1$. From the definition of $g_{1}$, we find, after a short calculation, that

$$
\begin{gathered}
g_{1}\left(z_{1}, z_{2}, \cdots, z_{N}\right)-g_{1}\left(z_{1}^{*}, z_{2}, \cdots, z_{N}\right) \\
=\left(\frac{1}{2 \pi}\right)^{N} \int_{-\pi}^{\pi} h\left(\varphi_{1}\right) P\left(z_{1}, \varphi_{1}\right) d \varphi_{1} \int \frac{2 u\left(e^{i \varphi_{1}}, e^{i \varphi_{2}}, \cdots, e^{i \varphi_{N}}\right)}{\left(1-z_{2} e^{-i \varphi_{2}}\right) \cdots\left(1-z_{N} e^{-i \varphi_{N}}\right)} d \varphi_{2} \cdots d \varphi_{N} .
\end{gathered}
$$

Here $P\left(z_{1}, \varphi_{1}\right)$ is the one dimensional Poisson kernel. The real part of the inner integral is bounded in $\varphi_{1}$, as may be seen from the fact that if we replace in the first part of our argument the function $h$ by the function identically 1 and then restrict the resulting function of $z_{1}, \cdots, z_{N}$ to the hyperplane $z_{1}=0$, our argument shows that the resulting function of $z_{2}, \cdots, z_{N}$ is uniformly bounded in $U^{N-1}$. Thus,

$$
\operatorname{Re}\left\{g_{1}\left(z_{1}, z_{2}, \cdots, z_{N}\right)-g_{1}\left(z_{1}^{*}, z_{2}, \cdots, z_{N}\right)\right\}
$$

is bounded, and the lemma is established.

II. The case of continuous boundary values, If $K$ is a set in $\mathrm{C}^{N}$, let $\mathscr{X}[K]$ denote the algebra of functions continuous on $\bar{K}$ and holomorphic in its interior. Similarly, if $E$ is a commutative Banach algebra with identity, let $\mathscr{A}[K, E]$ be the algebra of all continuous $E$-valued functions on $\bar{K}$ which are analytic in its interior. There are several formally different definitions of analytic $E$-valued functions which are in fact equivalent. For a discussion of these see [4]. For the sake of definiteness, let us say that F: $\Omega \rightarrow E, \Omega$ an open set in $\mathrm{C}^{N}$, is analytic if given $z^{0}=\left(z_{1}^{0}, \cdots, z_{N}^{0}\right) \varepsilon \Omega$, there is an expansion

$$
F(z)=\sum e_{j_{1} \cdots j_{N}}\left(z_{1}-z_{1}^{0}\right)^{j_{1}} \cdots\left(z_{N}-z_{N}^{0}\right)^{j_{N}}
$$

with coefficient $e_{j_{1}} \ldots j_{N}$ elements of $E$, where the summation extends over all $N$ tuples of nonnegative integers, and where $\sum\left\|e_{j_{1}} \cdots j_{N}\right\| \rho^{j_{1}+\cdots+j_{N}}$ is convergent for some $\rho>0$. The following theorem obtains.

THEOREM II.1. Let $\mathscr{y}^{-}=\left\{V_{\alpha}\right\}_{\alpha \in A}$ be an open covering of the closed unit polycylinder $\bar{U}^{N}$ in $\mathbf{C}^{N}$, and for each $\alpha$ let $f_{\alpha} \in \mathscr{A}\left[V_{\alpha}, E\right]$. If for all $\alpha, \beta \in A$ there exists $h_{\alpha_{\beta}}$, an invertible element of $\mathscr{A}\left[V_{\alpha} \cap V_{\beta}\right]$, such that on $\bar{V}_{\alpha} \cap \bar{V}_{\beta} f_{\alpha}=f_{\beta} h_{\alpha \beta}$, then there exists $F \in \mathscr{A}\left[\bar{U}^{N}, E\right]$ such that for all $\alpha \in A, F=f_{a} h_{\alpha}, h_{\alpha}$ an invertible element of $\mathscr{A}\left[V_{\alpha}, E\right]$.

1 This argument was suggested by W. Rudin as an alternative to a more complicated argument of the author. 
The proof of this result depends on the fact proved by Douady ${ }^{2)}$ [2, p. 48] that if $\Delta, \Delta^{+}$and $\Delta^{-}$are as in the proof of Theorem I.1, and if $F$ is an invertible element of $\mathscr{A}\left[\left(\Delta^{+} \cap \Delta^{-}\right), E\right]$ then there exist invertible $F_{+} \in \mathscr{A}\left[\Delta^{+}, E\right]$ and $F_{-} \in \mathscr{\mathscr { A }}\left[\Delta^{-}, E\right]$ such that on $\Delta^{+} \cap \Delta^{-}, F=F_{+} F_{-}$.

Once one has this fact, it is possible to argue by contradiction and establish Theorem II.1 just as Theorem I.1 was established.

As an application of Theorem II.1, we will show that certain sets in $T^{N}=\left\{z \in C^{N}:\left|z_{1}\right|=1, \cdots,\left|z_{N}\right|=1\right\}$ are zero sets for elements of $\mathscr{A}\left[U^{N}\right]$.

TheOREM II.2. Let $\beta_{1}, \cdots, \beta_{N}$ be strictly positive real numbers. Let $\Lambda$ be a compact set of Lebesgue measure zero in the real line, and let $G_{A}=\left\{t \in \boldsymbol{R}^{N}: t \cdot \beta=t_{1} \beta_{1}+\cdots+t_{N} \beta_{N} \in \Lambda\right\}$. If $E$ is a compact subset of $\left\{\left(e^{i t_{1}}, \cdots, e^{i t_{N}}\right): t \in G_{1}\right\}$, then $E$ is the zero set of an element of $\mathscr{A}\left[U^{N}\right]$.

Proof. Define a map $r: C^{N} \rightarrow C^{N}$ by means of $r\left(z_{1}, \cdots, z_{N}\right)=$ $\left(e^{i z_{1}}, \cdots, e^{i z_{N}}\right)$. Regarding $\boldsymbol{R}^{N}$ as the set of points in $\boldsymbol{C}^{N}$ with real coordinates, we have that $r$ carries $R^{N}$ onto $T^{N}$. If $Q_{+}^{N}$ denotes the set $\left\{z \in C^{N}: \operatorname{Im} z_{j} \geqq 0\right.$ for $\left.j=1, \cdots, N\right\}$, then $r$ carries $Q_{+}^{N}$ onto a dense subset of $\bar{U}^{N}$. The Jacobian of the map $\Upsilon$ is $\left.e^{i\left(z_{1}+\cdots+z_{N}\right.}\right)$ which never vanishes, so $r$ is a local homeomorphism at every point of $\boldsymbol{C}^{N}$.

Assume initially that there exists a compact set $K \subset G_{A}$ such that $r[K]=E$. In general no such set $K$ will exist, but we shall excise this difficulty later. If $t \in \boldsymbol{R}^{N}$, let

$$
\Delta(t, \varepsilon)=\left\{z \in Q_{+}^{N}:\left|t_{1}-z_{1}\right|<\varepsilon, \cdots,\left|t_{N}-z_{N}\right|<\varepsilon\right\} ;
$$

this is a certain product of half discs. For each $t^{0} \in K$, let $\varepsilon\left(t^{0}\right)$ be such that $r$ carries $\Delta\left(t^{0}, \varepsilon\left(t^{0}\right)\right)^{-}$homeomorphically onto a closed (relative) neighborhood $V\left(t^{0}\right)$ of the point $s^{0}=r\left(t^{0}\right)$. There is $\delta>0$ such that $\Delta^{\prime}\left(s^{0}, \delta\right)=\left\{z \in \bar{U}^{N}:\left|s_{1}^{0}-z_{1}\right|<\delta, \cdots,\left|s_{N}^{0}-z_{N}\right|<\delta\right\}$ is contained in $V\left(t^{0}\right)$.

Let $r_{t_{0}}^{-1}: V\left(t^{0}\right) \rightarrow \Delta\left(t^{0}, \varepsilon\left(t^{0}\right)\right)^{-}$be inverse to $r$. By compactness, finitely many of the sets $r_{t}^{-1}\left[\Delta^{\prime}\left(s^{0}, \delta / 2\right)\right]$ will cover $K$. Let the $\Delta^{\prime}\left(s^{0}, \delta / 2\right)$ corresponding to such a cover be $\Delta^{\prime}\left(s^{j}, \delta / 2\right), j=1, \cdots, q$, and let the corresponding $r_{t_{0}}^{-1}$ be $r_{j}^{-1}, j=1, \cdots, q$.

The set $\Lambda$ is a compact Lebesgue null set, so the Rudin-Carleson theorem [6, p. 81] applied to $Q_{+}$, the upper half plane, yields a function $F$ continuous on $\bar{Q}_{+}$, holomorphic on $Q_{+}$, which vanishes exactly on the set $\Lambda$. Define $H$ on $Q_{+}^{N}$ by $H(z)=F\left(\beta_{1} z_{1}+\cdots+\beta_{N} z_{N}\right)$. The zero set of $H$ is the set $G_{1}$.

Define $F_{j}$ on $A^{\prime}\left(s^{j}, \delta\right)$ by $f_{j}=H \circ r_{j}^{-1}$. The function $f_{j}$ is in

${ }^{2} \mathrm{I}$ am indebted to the referee for this reference. 
$\mathscr{A}\left[\Delta^{\prime}\left(s^{j}, \delta\right)\right]$ and vanishes on $r\left[K \cap \Delta\left(t^{j}, \varepsilon\left(t^{j}\right)\right)^{-}\right]$. Consequently [8], there is $g_{j} \in \mathscr{A}\left[\Delta^{\prime}\left(s^{j}, \delta\right)\right]$ which vanishes exactly on the part of $r\left[K \cap \Delta\left(t^{j}, \varepsilon\left(t^{j}\right)\right)^{-}\right]$which lies in $\Delta^{\prime}\left(s^{j}, \delta / 2\right)$.

Let $W_{j}=\left\{z \in \bar{U}^{N}\right.$ : for some $\left.k=1, \cdots, N,\left|z_{k}-s_{k}^{j}\right|>3 \delta / 4\right\}$. Let $\widetilde{g}_{j}$ be the function identically one on $\bar{W}_{j}$. Since on $W_{j} \cap \Delta^{\prime}\left(s^{j}, \delta\right), g_{j}$ is zero free, the functions $g_{j}$ and $\widetilde{g}_{j}$ constitute a set of Cousin II data to which Theorem II.1 can be applied. We conclude that there exists $F_{j} \in \mathscr{A}\left[U^{N}\right]$ which vanishes exactly on the part of $r\left[K \cap \Delta\left(t^{j}, \in\left(t^{j}\right)\right)^{-}\right]$which is contained in $\Delta^{\prime}\left(s^{j}, \delta / 2\right)$. Define $F$ to be the product of the finitely many functions $F_{j}$ so constructed. The function $F$ has $\gamma[K]=E$ as its zero set.

In the case that the set $D$ does not exist, we may write $E=\cup E_{k}$ where $E_{k}=\gamma\left[K_{k}\right], K_{k}$ a compact set in $G_{1}$. The remark on page 435 of [5] or the simpler Corollary 1.2 of [8] now implies that $E$ is the zero set of some element of $\mathscr{A}\left[U^{N}\right]$.

Let us note that in the light of Theorem 1.1 of [8], this theorem generalizes Theorem 4.6 of [7], the case that $\Lambda=\{0\}$.

It should be noted that Theorem II.2 is contained as a special case of more general results of Forelli [3] which are obtained by studying measures orthogonal to $\mathscr{A}\left[U^{N}\right]$.

Acknowledgement is due the very useful correspondence the author has had with Professor Walter Rudin on the subject of the present paper.

\section{REFERENCES}

1. L. Bers, Introduction to several complex variables, Courant Institute Lecture Notes, 1964.

2. A. Douady, Le problème des modules pour les sous-espaces analytiques compacts d'un espace analytique donné, Ann. Inst. Fourier XVI (1966), 1-95.

3. F. Forelli, Measures orthogonal to polydisc algebras, J. Math. Mech. 17 (1968), 1073-1086.

4. A. Gleason, The abstract theorem of Cauchy-Weil, Pacific J. Math. 12 (1962), 511-525.

5. I. Glicksberg, Measures orthogonal to algebras and sets of antisymmetry, Trans. Amer. Math. Soc. 105 (1962), 415-435.

6. K. Hoffman, Banach spaces of analytic functions, Prentice-Hall, Englewood Cliffs, N. J., 1962.

7. W. Rudin and E. L. Stout, Boundary properties of functions of several complex variables, J. Math. Mech. 14 (1965), 991-1006.

8. E. L. Stout, On some restriction algebras. Function algebras, edited by F. T. Birtel, Scott, Foresman, and Co., Chicago, 1966.

Received April 6, 1967. This work was supported, in part, by National Science Foundation Grant GP5493. 



\title{
PACIFIC JOURNAL OF MATHEMATICS
}

\author{
EDITORS
}

\author{
H. ROYDEN \\ Stanford University \\ Stanford, California
}

\author{
J. P. JANS \\ University of Washington \\ Seattle, Washington 98105
}

\section{J. DUGUNDJI}

Department of Mathematics University of Southern California Los Angeles, California 90007

\section{RICHARD ARENS}

University of California Los Angeles, California 90024

\section{ASSOCIATE EDITORS}

\author{
E. F. BECKENBACH
}

B. H. NEUMANN

F. WoLF

K. YosidA

\section{SUPPORTING INSTITUTIONS}

\author{
UNIVERSITY OF BRITISH COLUMBIA \\ CALIFORNIA INSTITUTE OF TECHNOLOGY \\ UNIVERSITY OF CALIFORNIA \\ MONTANA STATE UNIVERSITY \\ UNIVERSITY OF NEVADA \\ NEW MEXICO STATE UNIVERSITY \\ OREGON STATE UNIVERSITY \\ UNIVERSITY OF OREGON \\ OSAKA UNIVERSITY \\ UNIVERSITY OF SOUTHERN CALIFORNIA
}

\author{
STANFORD UNIVERSITY \\ UNIVERSITY OF TOKYO \\ UNIVERSITY OF UTAH \\ WASHINGTON STATE UNIVERSITY \\ UNIVERSITY OF WASHINGTON \\ AMERICAN MATHEMATICAL SOCIETY \\ CHEVRON RESEARCH CORPORATION \\ TRW SYSTEMS \\ NAVAL WEAPONS CENTER
}

Mathematical papers intended for publication in the Pacific Journal of Mathematics should be in typed form or offset-reproduced, double spaced with large margins. Underline Greek letters in red, German in green, and script in blue. The first paragraph or two must be capable of being used separately as a synopsis of the entire paper. It should not contain references to the bibliography. Manuscripts, in duplicate if possible, may be sent to any one of the four editors. All other communications to the editors should be addressed to the managing editor, Richard Arens, University of California, Los Angeles, California 90024.

Each author of each article receives 50 reprints free of charge; additional copies may be obtained at cost in multiples of 50 .

The Pacific Journal of Mathematics is published monthly. Effective with Volume 16 the price per volume (3 numbers) is $\$ 8.00$; single issues, $\$ 3.00$. Special price for current issues to individual faculty members of supporting institutions and to individual members of the American Mathematical Society: $\$ 4.00$ per volume; single issues $\$ 1.50$. Back numbers are available.

Subscriptions, orders for back numbers, and changes of address should be sent to Pacific Journal of Mathematics, 103 Highland Boulevard, Berkeley 8, California.

Printed at Kokusai Bunken Insatsusha (International Academic Printing Co., Ltd.), 7-17, Fujimi 2-chome, Chiyoda-ku, Tokyo, Japan.

PUBLISHED BY PACIFIC JOURNAL OF MATHEMATICS, A NON-PROFIT CORPORATION

The Supporting Institutions listed above contribute to the cost of publication of this Journal, but they are not owners of publishers and have no responsibility for its content or policies. 


\section{Pacific Journal of Mathematics}

Vol. 26, No. 2 December, 1968

Seymour Bachmuth and Horace Yomishi Mochizuki, Kostrikin's theorem on

Engel groups of prime power exponent ....................

Paul Richard Beesack and Krishna M. Das, Extensions of Opial's inequality ...................................... 215

John H. E. Cohn, Some quartic Diophantine equations .............. 233

H. P. Dikshit, Absolute $(C, 1) \cdot\left(N, p_{n}\right)$ summability of a Fourier series and its conjugate series ............................... 245

Raouf Doss, On measures with small transforms ................. 257

Charles L. Fefferman, $L_{p}$ spaces over finitely additive measures........ 265

Le Baron O. Ferguson, Uniform approximation by polynomials with integral coefficients. II...................................

Takashi Ito and Thomas I. Seidman, Bounded generators of linear

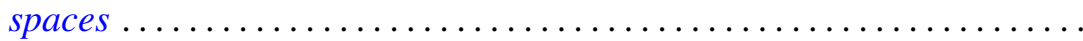

Masako Izumi and Shin-ichi Izumi, Nörlund summability of Fourier series ..........................................

Donald Gordon James, On Witt's theorem for unimodular quadratic

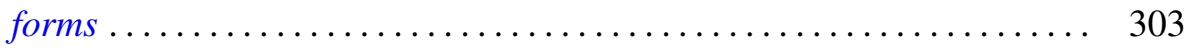

J. L. Kelley and Edwin Spanier, Euler characteristics .............. 317

Carl W. Kohls and Lawrence James Lardy, Some ring extensions with matrix

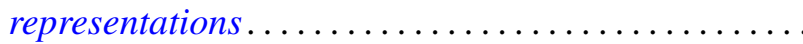

Ray Mines, III, A family of functors defined on generalized primary groups ....

Louise Arakelian Raphael, A characterization of integral operators on the space of Borel measurable functions bounded with respect to a weight function....

Charles Albert Ryavec, The addition of residue classes modulo $n .$.

H. M. (Hari Mohan) Srivastava, Fractional integration and inversion formulae associated with the generalized Whittaker transform ...

Edgar Lee Stout, The second Cousin problem with bounded data ...

Donald Curtis Taylor, A generalized Fatou theorem for Banach

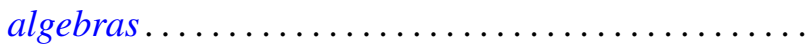

Bui An Ton, Boundary value problems for elliptic convolution equations of Wiener-Hopf type in a bounded region...

Philip C. Tonne, Bounded series and Hausdorff matrices for absolutely convergent sequences... 